\title{
Design of a Modular Swift-shift Multi-speed Transmission with Double Dual Clutches for Electric Vehicles
}

\author{
Alexei Morozov ${ }^{1}$, Ting Zou ${ }^{1}$, Mir Saman Rahimi Mousavi ${ }^{2}$, Jorge Angeles ${ }^{1}$, and Benoit Boulet ${ }^{2}$ \\ ${ }^{1}$ Centre for Intelligent Machines \& Department of Mechanical Engineering, McGill University, Montreal, \\ H3A0C3 Canada,Email: alexvit@cim.mcgill.ca,ting@cim.mcgill.ca, angeles@cim.mcgill.ca \\ ${ }^{2}$ Centre for Intelligent Machines \& Department of Electrical Engineering, McGill University, Montreal, \\ H3A2T5 Canada,Email: saman@cim.mcgill.ca,boulet@cim.mcgill.ca
}

\begin{abstract}
In this paper a novel concept of modular swift-shift multi-speed transmission with double dual clutches is proposed, with its architecture, simulation and control strategies discussed in detail. Due to its modularity, the desired number of gear ratios can be readily realized by adding the appropriate number of modules. Further, in this design, both the underdrive and the overdrive modes are combined within one single gear train, which reduces the overall volume and weight significantly. Due to its modularity and compactness, this transmission is suitable for electric vehicles, especially commercial fleets of medium- and heavy-duty electric trucks. A proof-of-concept testbed was developed; its preliminary simulation analysis is reported here.
\end{abstract}

Keywords: swift-shift, multi-speed transmission, double dual-clutch, Simdriveline, electric vehicles

\section{INTRODUCTION}

The idea of using electric and hybrid technologies for medium and heavy trucks in recent years became quite popular among automotive manufacturers; one of the potential sales categories for emerging hybrid and batterypowered vehicles includes commercial delivery fleets.

Currently the market of electrified trucks can be split into three categories of vehicles: delivery trucks, city (service) trucks, and e-buses. Generally, life-cycle vehicle cost consists of three main components: initial cost, ownership cost and recycling cost. High manufacturing cost of battery-motor systems is the main reason for the initial high cost of HEVs [1]. On average, hybrid vehicles cost 15-20\% higher than their identical gasoline counterparts. However, in terms of fuel economy, the mile per gallon (mpg) parameter for HEV can be two-three times bigger than that of conventional vehicles [2]. Besides, battery replacement is the unique additional cost of HEVs, but with the development of the technology, the influence of this factor will obviously diminish. For delivery trucks the hybrid option is economically efficient only in low mileage and high stop-and-go driving cycles. Though the maintenance cost per $\mathrm{km}$ of a hybrid truck can be close to that in conventional trucks, the annual maintenance expenditure remains lower since fuel economy makes sense in these driving cycles only, the hybrid truck staying in service in such driving cycles. In turn, the gas truck in high stop-and-go usage costs more both in fuel and maintenance due to higher cost of brake service. Gas trucks do not have regeneration braking, while being subject to more intensive brake wear. All these issues, along with the longer oil change intervals in hybrids (due to the engine running less), make the benefits of hybrid delivery trucks apparent. For example, research undertaken between 2008 and 2011 for the Purolator delivery fleet showed that the average costs of maintenance per $\mathrm{km}$ for both gas and hybrid trucks are close to each other (both within the range of $\$ 0.15-\$ 0.20 / \mathrm{km}$ ), while the maintenance cost of a hybrid is nearly 30 percent lower than that of a gas unit (app. \$2700 vs. \$4000). 
Series hybrid-diesel heavy trucks are being developed not only for commercial applications. As recently as 2002 , Oshkosh Truck Corporation introduced the first in the world electric hybrid-drive Heavy Expanded Mobility Tactical Truck HEMTT-LHS featuring Oshkosh's ProPulse electric hybrid-drive propulsion system [3]. According to the company expectations, fuel economy could reach $40 \%$. Eventually, OSHKOSH Defence successfully implemented the ProPulse diesel electric drive system in the mass production of military Heavy Expanded Mobility Tactical Truck (HEMTT) A3 (first disclosed at CALSTART's Hybrid Truck Users Forum HTUF in Atlanta in October 2009). However, the actual improvement of fuel economy reaches only 20\% [4], which is lower than the expected amount, but still quite good.

In a survey of medium and heavy hybrid trucks it is noteworthy that the biggest hybrid truck in the world [5] is developed for mining applications by Siemens and is powered by an electric drive system consisting of four electric motors, each of $1200 \mathrm{~kW}$, capable of moving the total weight of a loaded truck of 800 ton.

After hybridization the next step in the application of electric technology to urban, delivery and service fleets is the development of all-electric, or battery-electric, commercial vehicles. Most recent research results [6] predict that battery-electric commercial vehicles in urban fleets will boom, and worldwide sales of electric drive and electricassisted commercial vehicles will grow tenfold between 2014 and 2023, reaching nearly 160,000 units per year. Lyden [7] analyzes the prospects of all-electric medium-duty (Class 3-7) trucks in modern business for the sake of elimination of fossil fuel and achieving zero direct emissions. This will happen due to the main advantages of all-electric trucks, namely, fuel cost savings, lower maintenance costs and increased productivity, which by 2020 will bring the number of e-trucks in the US market close to 50 percent of the total medium truck fleet. Needless to say, that, along with the hybrid versions of medium trucks, the total number of electrical commercial vehicles is expected to become the dominant majority in the market.

In the discussion of the current trends in the market of medium battery-electric trucks one can mention the series of prototypes developed by EMOSS Hybrid \& Electric drive systems, such as 10-, 12-, 16- and 18-tonne electric trucks ${ }^{1}$. The biggest of them reaches a top speed of $85 \mathrm{~km} / \mathrm{h}$, with a range up to $230 \mathrm{~km}$.

For medium electric trucks the most critical components are the batteries and the traction motors, which should have enough power to start and run the vehicle. For heavy trucks this problem is even more serious.

Regarding batteries, those that have enough power to drive a truck are quite heavy, which poses a real challenge for designers in selecting the appropriate batteries. In order to solve the foregoing problems, engineers suggest different creative solutions, e.g., the series of EMOSS trucks with configurable battery packs $(40,80,120,160$, 200 and $300 \mathrm{kWh}$ ), with which the vehicles can achieve a range ${ }^{2}$ from 25 to $300 \mathrm{~km}$.

Since the issue of battery size and weight is critical for the application in medium and heavy trucks, a related problem arises: packaging and space reduction. If the battery volume cannot be reduced, then other approaches should be used. One such approach is the reduction of the powertrain size, namely, transmission and final drive. The use of transmissions for electric vehicles is an important topic of modern research activities in the EV market. For example, in 2012, one of the world leaders in transmission technology, Oerlikon Graziano, in collaboration with Vocis Driveline Control, reported the results of a case study on a two-speed system for EVs [8]. These results clearly demonstrated, as confirmed by many other research teams $[9,10]$, the advantages of the two-speed transmission when compared to a single-speed model used in EVs via four main characteristics: acceleration time; maximum climbing grade; maximum speed; and energy consumption.

However, most current developments explore the application of multi-speed (mostly, two-speed) transmissions only in the passenger- and sports-car EV segment, whereas the more powerful the powertrain, the larger the number of gears in the transmission should be. Among very few prototypes of e-trucks supplied with a transmission, the EMOSS $150 \mathrm{~kW}$ Powertrain can be mentioned with three transmission speeds: 1:6.4, 1:8 and 1:10.2.

The authors of this paper conducted research on the use of two-speed transmissions in class-4 electric delivery medium-duty trucks [11], where they found that, contrary to small passenger cars, the use of a two-speed transmission in medium-duty e-trucks does not affect efficiency, but significantly improves performance, in comparison with direct drive battery e-trucks: gradeability and maximum speed are higher, while acceleration time is reduced. While energy consumption for class-4 e-trucks does not change much, using of a two-speed powertrain makes it possible to improve packaging by reducing the size of the traction motor and inverter, and, consequently, their cost. Though the transmission brings-in its own weight and cost, our estimations show that, in combination with the savings of the motor, the total weight reduction of the complete powertrain may reach up to four times, and up to twice the cost, in comparison with the direct drive option. Further research $[12,13]$ confirmed previous conclusions drawn by the authors.

A novel modular concept of multi-speed transmission for the electric vehicles was proposed by the authors of this paper [14], which allows for simple transmissions with virtually any required number of gears and seamless gearshifting. The transmission consists of two separate gear trains - the underdrive and the overdrive-with common

\footnotetext{
${ }^{1}$ EMOSS Hybrid \& Electric Drive Systems, e-Powertrain/Full electric powertrain, http://www.emoss.biz/electric-powertrain.
}

${ }^{2}$ EMOSS Hybrid \& Electric Drive Systems, e-Truck /Full electric truck, http://www.emoss.biz/electric-truck. 
planet carrier and with the number of planetary gear modules in each gear train corresponding to the number of required gears in each mode.

In this paper the concept is developed further by means of a compact version of the multi-speed transmission, with merely half the number of planetary gear sets than reported earlier [14], and two dual clutches. The reduced size of the transmission is especially important for heavy trucks carrying large batteries. Being automatic, the transmission nevertheless does not have a torque converter, since the motor is fully controlled in start, drive or reverse operations.

\section{CONCEPT DESCRIPTION}

In the proposed concept modularity is the most important feature. To obtain the required number of gears, the transmission can be designed by simply assembling the appropriate number of modules. Each module is based on a planetary gear set and, in combination with clutches, the stack of modules provides smooth driveability and surgeless swift-shift capability. Contrary to the earlier transmission [14], the new design has only one gear train that produces both the underdrive and the overdrive speed ratios. Each module generates two ratios; for each additional pair of underdrive/overdrive ratios we need only one additional module. This concept makes the transmission lighter and more compact.

\subsection{Transmission gear train}

The gear train of the proposed transmission is shown in Fig. 1. This transmission can be built with the desired number of steps, $n$, each step providing two speed modes, i.e., the underdrive and the overdrive ratios are paired and their total numbers in this transmission are equal.

The concept is based on the series of $n$ planetary gear sets (modules). All planets are installed on a common planet carrier PC, each axis of the latter carrying a group of $n$ planets $\mathrm{P}_{1}, \mathrm{P}_{2}, \cdots, \mathrm{P}_{n}$, each planet being coupled with the corresponding sun gear. All sun gears are rigidly fixed to a common shaft. The tooth surface of the planet with the smallest diameter $\mathrm{P}_{1}$ is in contact with the tooth surface of the biggest sun $\mathrm{S}_{1}$, and the tooth surface of the planet with the biggest diameter $\mathrm{P}_{n}$ is in contact with the tooth surface of the smallest sun $\mathrm{S}_{n}$.

The gear train also includes $n$ ring gears $\mathrm{R}_{1}, \mathrm{R}_{2}, \cdots, \mathrm{R}_{n}$ of different pitch diameters, matching the dimensions of corresponding planet and sun gears.

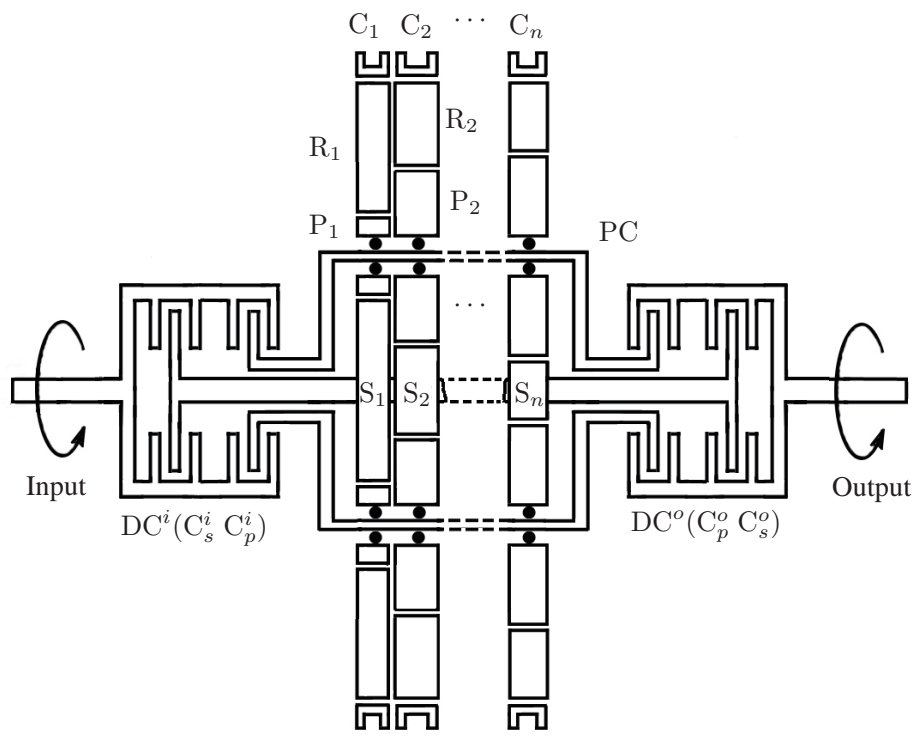

Figure 1: Multi-speed transmission layout

The input rotation can be translated through a transmission by several alternative ways. The key elements in redirection of the power flow are two dual clutches, one located at the input of the transmission, the other at the output. The first dual clutch $\mathrm{DC}^{i}$ connects the input shaft of the transmission with the shaft of the sun gears or with the input shaft of the planet carrier PC. The second dual-clutch $\mathrm{DC}^{o}$ connects the shaft of the sun gears or the output shaft of PC with the output shaft of the transmission. 


\subsection{Modes of operation}

\subsubsection{Underdrive mode}

The rotation from the traction motor is transmitted from the input transmission shaft to the sun gears. For this, the input clutch $\mathrm{C}_{s}^{i}$ of the input dual clutch $\mathrm{DC}^{i}$ is engaged and connects the input transmission shaft with the input of the sun shaft. The clutch $\mathrm{C}_{p}^{i}$ is released. The input torque and rotation are transmitted from a sun to one of the planets. The planet is selected by engagement of the corresponding friction clutch $\mathrm{C}_{k}$ (where $k=1, \cdots, n$ ), according to the control algorithm. The engaged friction clutch immobilizes the corresponding ring gear $\mathrm{R}_{k}$. All other friction clutches are released, and the torque/rotation is transmitted via the planet $\mathrm{P}_{k}$ to the planet carrier. The output rotation of the transmission is taken from the rotation of the planet carrier with the help of the clutch $\mathrm{C}_{p}^{o}$ of the second dual clutch $\mathrm{DC}^{o}$, which engages the planet carrier with the output transmission shaft.

In this mode the output speed of the transmission is lower than that of the input shaft. The ratio of the speed reduction depends on the choice of the ring gear fixed to the housing by the corresponding friction clutch. Totally, the transmission has $\mathrm{n}$ different ratios in the underdrive mode.

\subsubsection{Overdrive mode}

The input rotation is transmitted from the input shaft to the planet carrier. For this, the input clutch $\mathrm{C}_{p}^{i}$ of the input dual clutch $\mathrm{DC}^{i}$ is engaged and connects the input transmission shaft with the input shaft of the planet carrier. The clutch $\mathrm{C}_{s}^{i}$ is released. The input torque and rotation are transmitted from the planet carrier via one of the planets to a corresponding sun gear. The planet is selected by engagement of the corresponding friction clutch $\mathrm{C}_{k}$, according to the control algorithm. The engaged friction clutch immobilizes the corresponding ring gear $\mathrm{R}_{k}$. All other friction clutches are released, and the torque/rotation is transmitted via the planet $\mathrm{P}_{k}$ to the sun $\mathrm{S}_{k}$. The output rotation of the transmission is taken from the rotation of the sun shaft with the help of the clutch $\mathrm{C}_{s}^{o}$ of the second dual clutch $\mathrm{DC}^{o}$, which engages the sun shaft with the output transmission shaft.

In this mode the output speed of the transmission is higher than that of the input shaft. The ratio of the speed increment depends on the choice of the ring gear fixed to the housing by the corresponding friction clutch. Totally, the transmission has $n$ different ratios in the overdrive mode.

As can be seen, the proposed transmission has the same number of underdrive and overdrive ratios. The value $n$ can be selected by the designer, based on the required specification for the vehicle driveline.

\subsubsection{Direct drive, reverse and neutral modes}

If both clutches $\mathrm{C}_{s}^{i}$ and $\mathrm{C}_{p}^{i}$ of the first dual clutch $\mathrm{DC}^{i}$, and both clutches $\mathrm{C}_{p}^{o}$ and $\mathrm{C}_{s}^{o}$ of the second dual clutch $\mathrm{DC}^{o}$ are engaged, and all $n$ friction clutches $\mathrm{C}_{1}, \mathrm{C}_{2}, \cdots, \mathrm{C}_{n}$ are released, the input speed and torque of the transmission are translated directly to its output shaft without any changes. This leads to the direct-drive mode of the transmission. The same arrangement provides transmission of the reversed input rotation to the reversed output rotation, in case of the reverse work mode of the motor.

In the direct drive and reverse modes, all components-the sun gears, the planet carrier, the planets, and the ring gears-rotate together, in a single block, at the same speed. There is no relative motion between the sun gears, the planets and the ring gears.

The total number of speeds in the proposed transmission, including direct drive, is

$$
k=2 n+1
$$

The neutral mode is provided when all clutches of both dual-clutches $\mathrm{DC}^{i}$ and $\mathrm{DC}^{o}$ are released, and no input rotation is transmitted to the output shaft. As opposed to the multi-speed transmission proposed by the same team in [14], this transmission does not have any intermediate ratios.

\section{TESTBED DESIGN AND PARAMETERS}

In order to validate the performance of the proposed multi-speed transmission with double dual clutches for electric vehicles, the testbed, shown in Fig. 2, was developed at McGill University's Centre for Intelligent Machines.

The scaled-down proof-of-concept testbed has a gear train, with two planetary gear sets, which incorporates both underdrive and overdrive modes into the same gearbox using double dual clutches to generate two power transmission routes (Fig. 3). This allows for four gears to be selected for power transmission through to the wheels, where each planetary gear set counts for two speeds-one overdrive and one underdrive. As well, there is a direct-drive mode that serves as Gear 3 among others. Therefore, this testbed has a total of five speeds. 


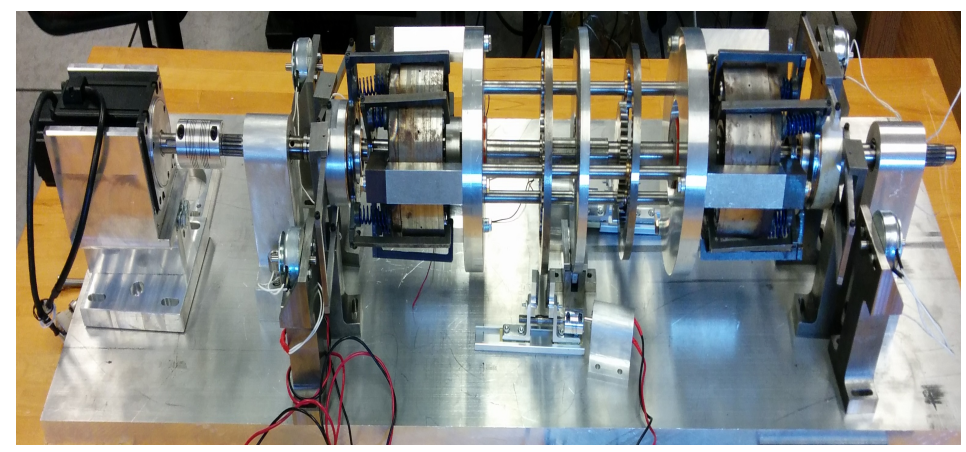

Figure 2: Testbed of the five-speed transmission with double dual-clutches

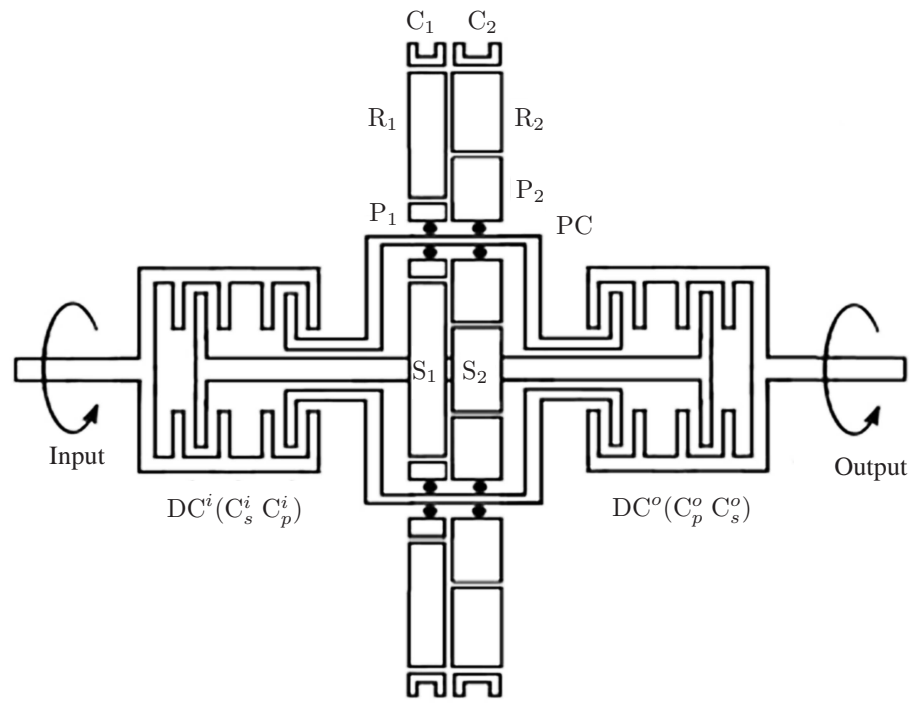

Figure 3: Layout of the five-speed testbed of the multi-speed transmission with double dual-clutches

The gear ratios of the transmission modules in the underdrive mode are $4: 1$ and $2.67: 1$; in the overdrive mode, $1: 2.67$ and $1: 4$.

For the proof-of-concept testbed, a high bandwidth Pulse-Width Modulation (PWM) brushless servo-amplified Glentek Motor "GMBM80550-45" was used ${ }^{3}$.

\section{SIMULATION RESULTS AND MODEL VERIFICATION}

\subsection{Multi-speed transmission system with constant torque input}

Figure 4 illustrates a simplified simulation model of the powertrain of an electric vehicle with the proposed fivespeed transmission, using Matlab/Simdriveline, to evaluate the gear shifting performance. A constant torque of $\tau_{i}=100 \mathrm{~N} \mathrm{~m}$ is applied to the input end of the model. The detailed layout for the five-speed transmission with a double dual-clutch subsystem is illustrated in Fig. 5.

As described in Sec. 3, the devised upshifting process was implemented in the simulation, with the gear ratios and clutch states listed in Table 1.

The input and output angular velocities of the five-speed transmission are plotted in Fig. 6, from which a smooth transition from rest to ascending speed can be observed. It is noteworthy that in this preliminary simulation test the input acceleration torque was constant, since our goal was to estimate the transition effect during shifting and not the actual gear shifting schedule. Figures 7(a) and (b) illustrate the normalized clutch pressure signals and transmission ratios during the upshifting process, respectively, which tally with the clutch schedule listed in Table 1.

${ }^{3}$ Motor parameters can be found through http: / / www . glentek. com/gmbmsel . aspx. 


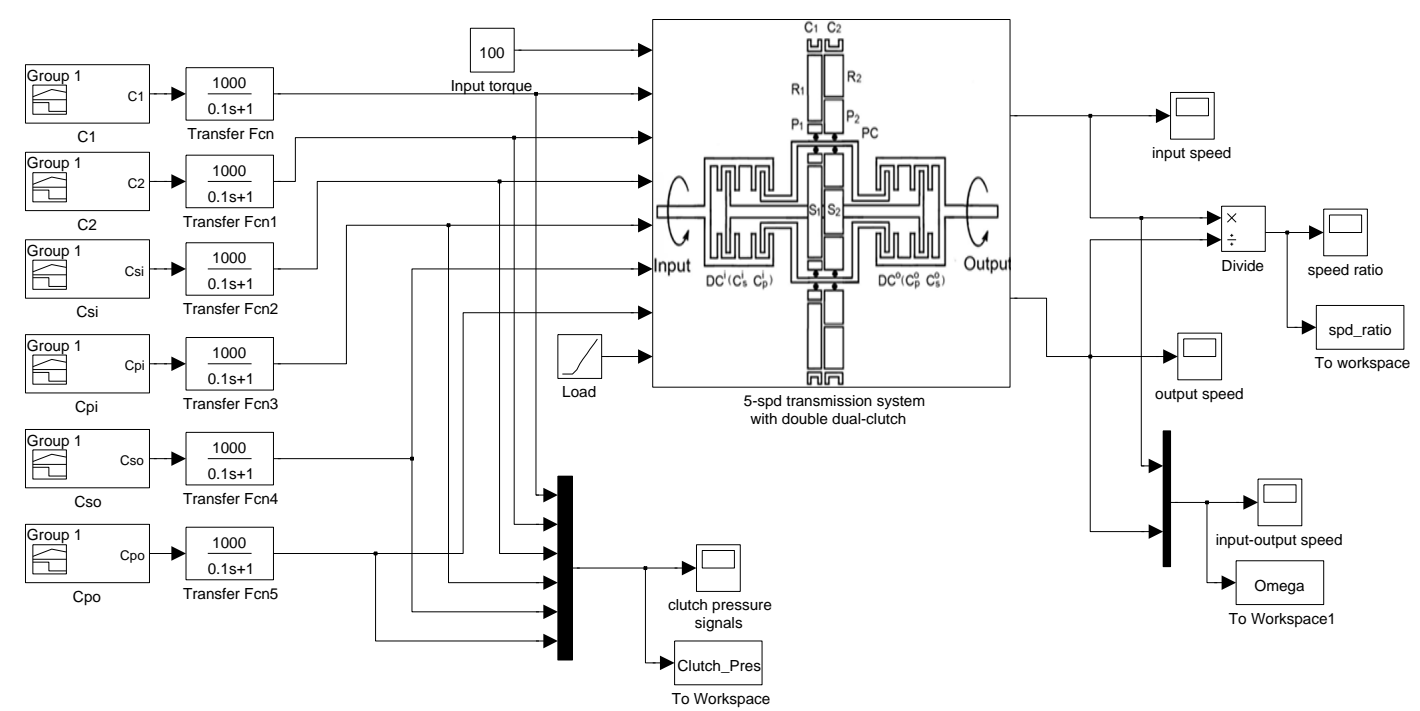

Figure 4: SimDriveline model of an electric vehicle powertrain equipped with the proposed 5-speed transmission $\left(\tau_{i}=\right.$ $100 \mathrm{~N} \mathrm{~m})$

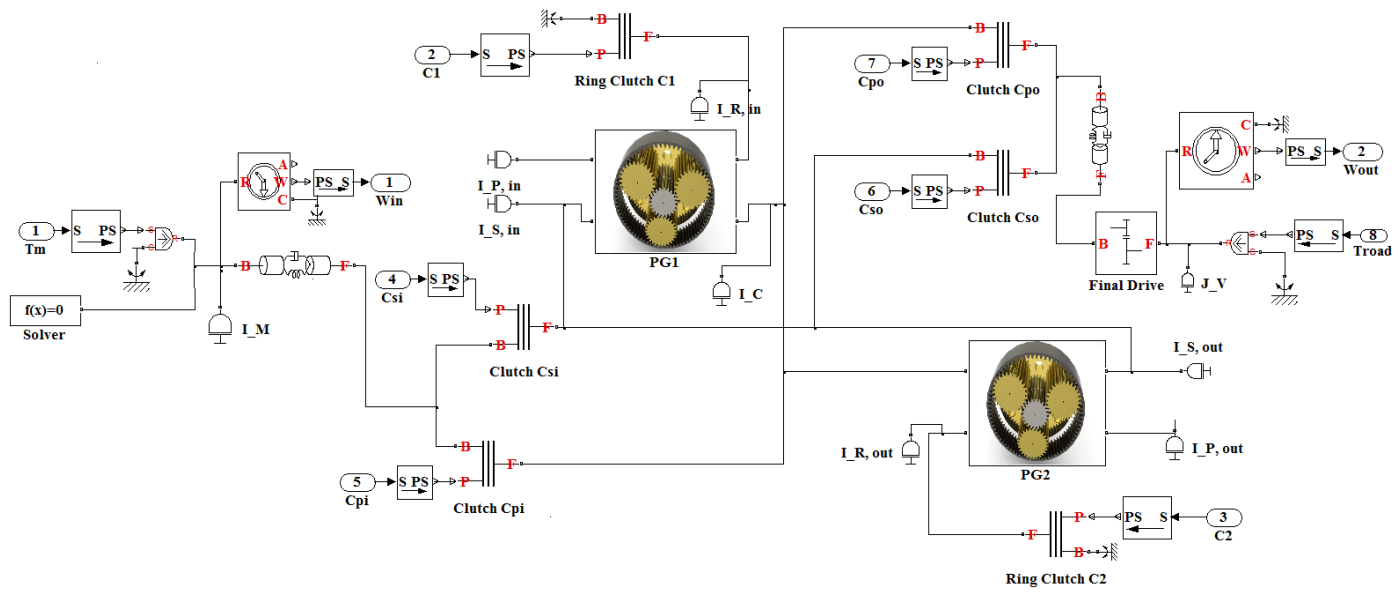

Figure 5: Layout of the 5-speed transmission with double dual-clutch

From Fig. 7(b), it is apparent that vibration occurs during the gear shifting periods, especially at the beginning of the simulation and during the transition from the first gear ratio to the second one. The main reason lies in that the constant input torque signal is not capable of simulating the actual gear shifting process for the vehicle precisely. Hence, to better evaluate the gear shifting performance, a simulation test of the five-speed transmission combined with a complete vehicle model was carried out, as discussed below.

\subsection{Multi-speed transmission system with the complete vehicle model}

As shown in Fig. 8, the proposed five-speed transmission was further tested with a complete vehicle model, including the engine, the transmission, as well as the vehicle and tire dynamics. The vehicle parameters are listed in Table $2^{4}$. The preprogrammed clutch signal schedule is plotted in Fig. 9(a). With reference to Table 1, we can observe that the preprogrammed transmission controller aims to implement an upshifting process, similar to the constant-input-torque example discussed earlier. Specifically, the vehicle will start with the first underdrive mode, i.e., with a gear ratio of 4 ; then it will shift to the second underdrive, neutral, the first overdrive and the second overdrive modes, consecutively, at $10 \mathrm{~s}, 35 \mathrm{~s}, 55 \mathrm{~s}$ and $75 \mathrm{~s}$, respectively.

The vehicle velocity obtained is shown in Fig. 9(b). Besides, the plots for the input and the output angular velocity of the transmission system are illustrated in Fig. 10(a). In Fig. 9(b), one can observe "small" peaks on the vehicle

${ }^{4}$ The parameters are taken from the Matlab/SimDriveLine demo "sdl_vehicle." 


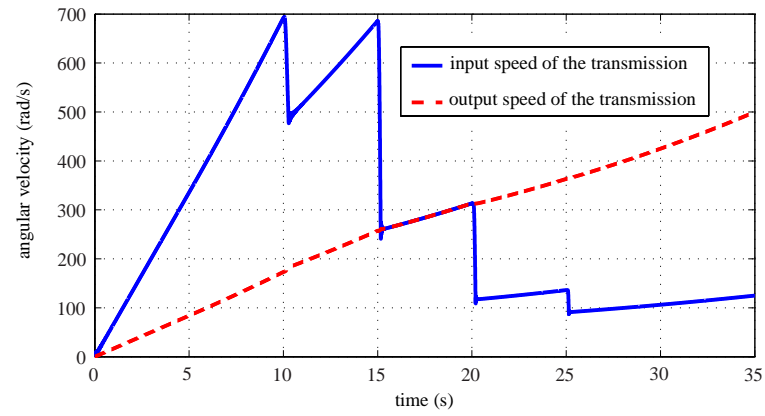

Figure 6: Input and output angular velocities of the transmission $\left(\tau_{i}=100 \mathrm{~N} \mathrm{~m}\right)$

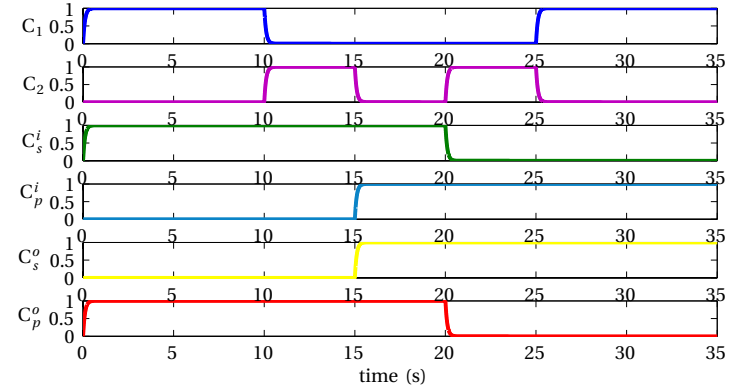

(a)

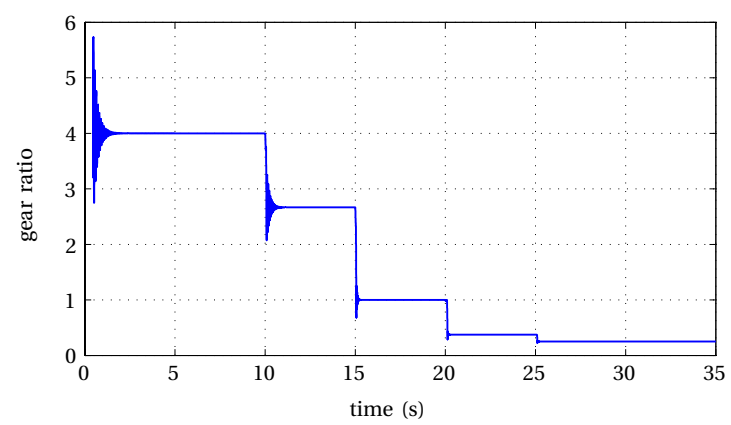

(b)

Figure 7: (a) Normalized clutch pressure signals; and (b) Simulated gear ratios based on the actual testbed geometry $\left(\tau_{i}=\right.$ $100 \mathrm{~N} \mathrm{~m})$

speed plot, which occur due to the gear-shifting operations. Further, the corresponding transmission ratios, calculated as the ratio between the input and output angular velocities of the transmission, are shown in Fig. 10(b). We can observe that the transmission ratios obtained tally with the preprogrammed clutch-signal schedule. Moreover, the smoothness over the whole gear-shifting process for the complete vehicle model was satisfactory, in comparison with the vibrations shown in Fig. 7(b).

Furthermore, for the purpose of better imitating the automatic transmission controller in the actual vehicle, the stateflow chart, as shown in Fig. 11, is used to define the control logic, for both upshifting and downshifting processes. The layout of the simulation model, including the automatic controller, is shown in Fig. 12.

For the transmission controller in Fig. 8, the clutch schedule is preprogrammed, i.e., the duration time for each transmission mode is prescribed, as shown earlier in this Subsection. Hence, the current vehicle velocity will not have any effect on the gear-shifting process. On the contrary, the controller in Fig. 12 is capable of realizing the real-time control for gear-shifting, in terms of the feedback from the current vehicle velocity. Hence, a real-time interaction between the transmission controller and the vehicle velocity leads to a more realistic vehicle operation.

The vehicle velocity and calculated gear ratios are plotted in Figs. 13 and 14(a), respectively. It can be seen that the

Table 1: Clutch schedule for the 5-speed transmission with double dual-clutch

\begin{tabular}{c|c|c|c|c|c|c|c}
\hline \multirow{2}{*}{ Transmission state } & \multirow{2}{*}{ Gear ratio } & \multicolumn{7}{|c}{ Clutch state $^{1}$} \\
\cline { 3 - 9 } & & $\mathrm{C}_{1}$ & $\mathrm{C}_{2}$ & $\mathrm{C}_{s}^{i}$ & $\mathrm{C}_{p}^{i}$ & $\mathrm{C}_{s}^{o}$ & $\mathrm{C}_{p}^{o}$ \\
\hline underdrive & 4 & 1 & 0 & 1 & 0 & 0 & 1 \\
\hline underdrive & 2.67 & 0 & 1 & 1 & 0 & 0 & 1 \\
\hline neutral & 1 & 0 & 0 & 1 & 1 & 1 & 1 \\
\hline overdrive & 0.3745 & 0 & 1 & 0 & 1 & 1 & 0 \\
\hline overdrive & 0.25 & 1 & 0 & 0 & 1 & 1 & 0 \\
\hline
\end{tabular}

${ }^{1}$ The clutch states 0 and 1 denote disengaged and engaged, respectively. 


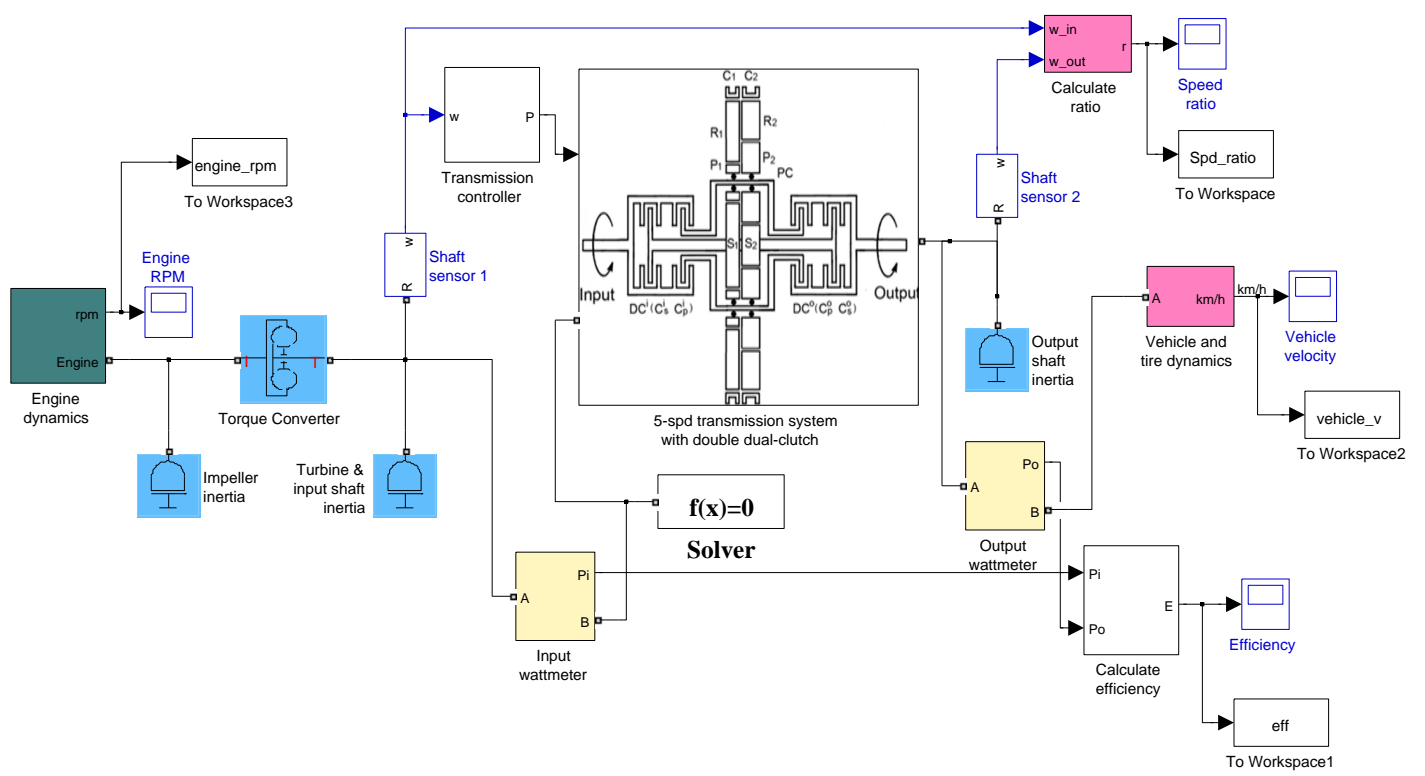

Figure 8: Simulation model of a complete vehicle with the proposed 5-speed transmission system

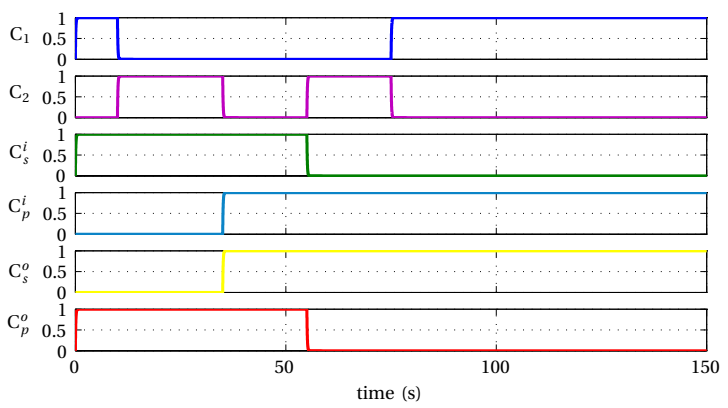

(a)

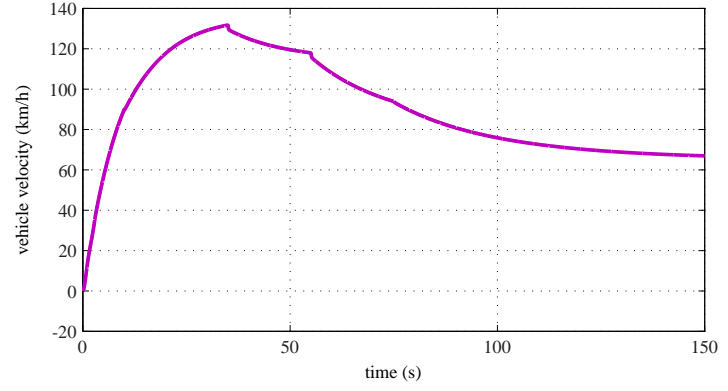

(b)

Figure 9: (a) Normalized clutch pressure signals; and (b) Vehicle velocity from the complete vehicle model

vehicle starts with a transmission ratio of 4 . When the vehicle speed increases to $25 \mathrm{~km} / \mathrm{h}$ at around $1.4 \mathrm{~s}$, it shifts to the ratio 2.67. Then, at $2.6 \mathrm{~s}$, the transmission shifts to a ratio of unity, i.e., direct drive mode, since the vehicle speed exceeds $45 \mathrm{~km} / \mathrm{h}$. The vehicle continues to accelerate. At around $5.5 \mathrm{~s}$, the vehicle speed reaches $60 \mathrm{~km} / \mathrm{h}$, the transmission thus shifting to the ratio 0.3745 from the direct drive mode. After that, the vehicle speed continues to increase. When the vehicle speed reaches $80 \mathrm{~km} / \mathrm{h}$, at round $18.7 \mathrm{~s}$, the vehicle shifts to the last transmission ratio, i.e., 0.25. Apparently, the simulation test shows an upshifting process, with all five speed ratios involved. During this test, the maximum velocity that the vehicle can reach is $94.96 \mathrm{~km} / \mathrm{h}$. It is apparent that the shifting process follows faithfully the control logic in Fig. 12. Moreover, this also can be validated by the clutch pressure signals obtained, as illustrated in Fig. 14(b).

Table 2: Vehicle parameters

\begin{tabular}{l|l|l}
\hline parameter & unit & value \\
\hline \hline mass & $\mathrm{kg}$ & 1200 \\
\hline Horizontal distance from center of gravity (CG) to front axle & $\mathrm{m}$ & 1.4 \\
\hline Horizontal distance from CG to rear axle & $\mathrm{m}$ & 1.6 \\
\hline CG height above ground & $\mathrm{m}$ & 0.5 \\
\hline Frontal area & $\mathrm{m}^{2}$ & 3 \\
\hline Drag coefficient & - & 0.4 \\
\hline
\end{tabular}




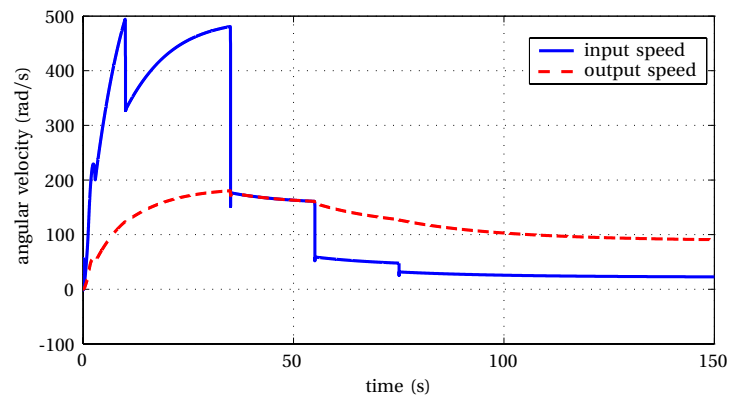

(a)

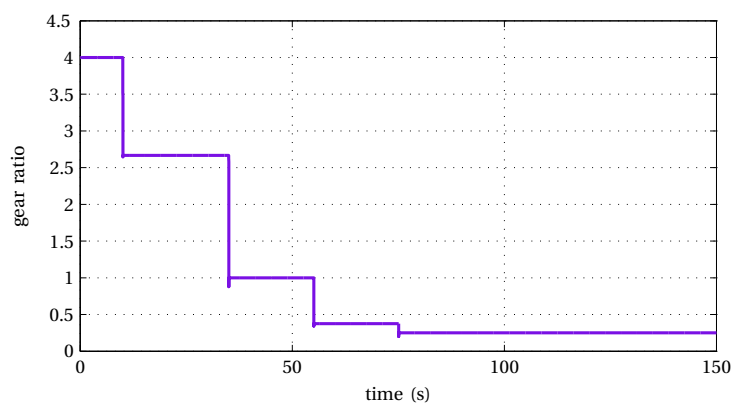

(b)

Figure 10: (a) Input and output angular velocities of the transmission; and (b) Speed ratio from the complete vehicle model

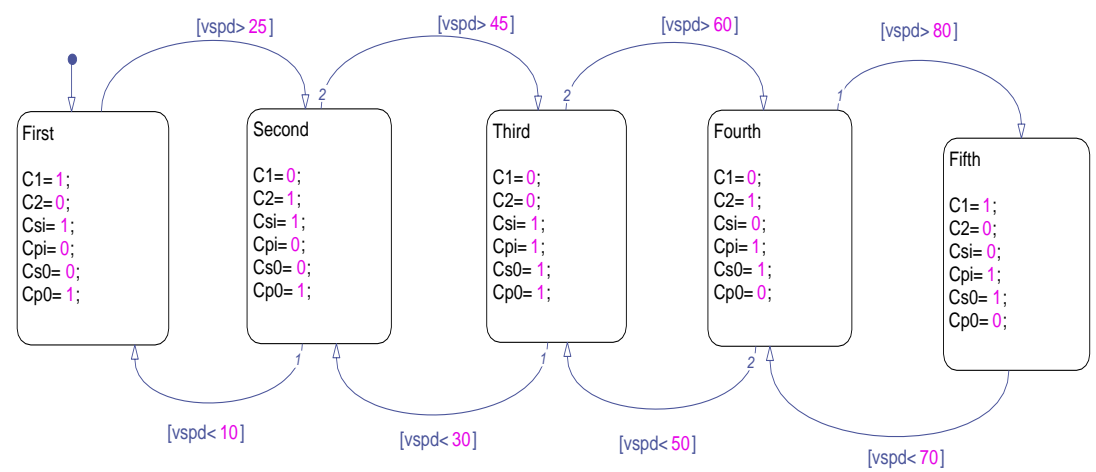

Figure 11: Stateflow chart of the clutch schedule for the transmission controller

\section{CONCLUSIONS}

The transmission of a modular type was proposed for use in electric vehicles of all sizes. Depending on class, power rating and required number of speed ratios, the transmission can be readily redesigned and modified by adding or removing extra planetary modules. Each module has two possible inputs and two possible outputs. Their serial combination makes up the full transmission, where the speed is smoothly controlled via the ring gears with the help of friction clutches, and by the smooth control of dual clutches installed at the input and the output of the transmission, as demonstrated in the presented simulation.

Due to the modular origins of the transmission and to the simplicity of the modules, its size and number of speeds can be readily modified and adjusted in the design process, depending on the required vehicle parameters. Because of modularity, such transmission is cheaper for production, manufacturing facilities readily adjustable to various transmission sizes. The transmission discussed is more compact than its predecessors [14], since the underdrive and the overdrive ratios are combined into pairs in corresponding modules. Therefore, the transmission itself is cheaper for manufacturing, smaller and easier for integration. Manufacturing facilities can be readily adjusted to various transmission sizes.

\section{ACKNOWLEDGMENTS}

The design and research work reported here was supported by Automotive Partnerships Canada grant APCPJ41890111.

We also gratefully acknowledge the support of our industrial partners: Linamar, TM4 and Infolytica.

\section{References}

[1] C. Lin, T. Wu, X. Ou, Q. Zhang, X. Zhang, and X. Zhang, "Life-cycle private costs of hybrid electric vehicles in the current chinese market," Energy Policy, vol. 55, pp. 501-510, 2013.

[2] T. Lipman and M. Delucchi, "A retail and lifecycle cost analysis of hybrid electric vehicles," Transportation Research Part D, vol. 11, pp. 115-132, 2006. 


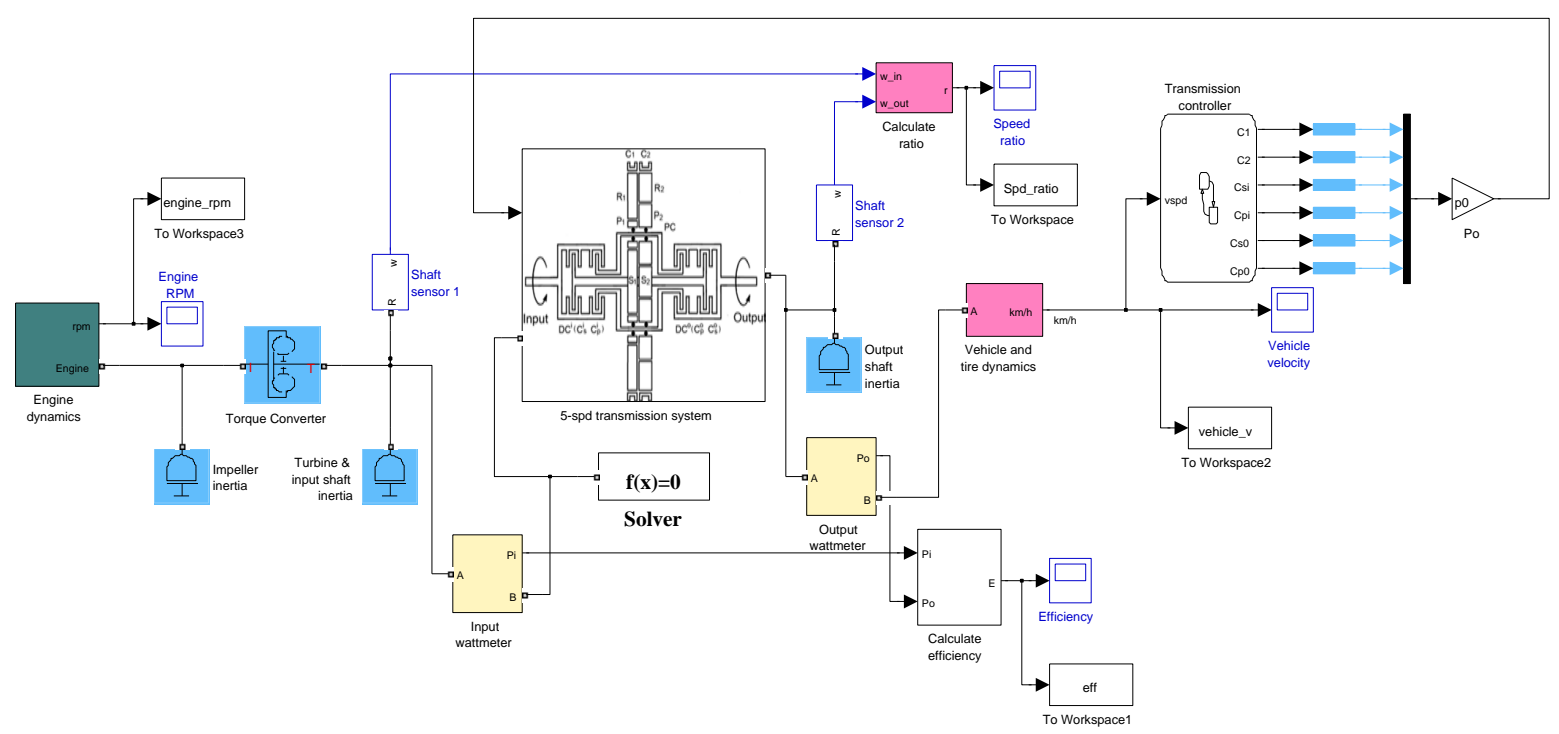

Figure 12: Simulation model of a complete vehicle with the proposed 5-speed transmission system integrated with automatic transmission controller

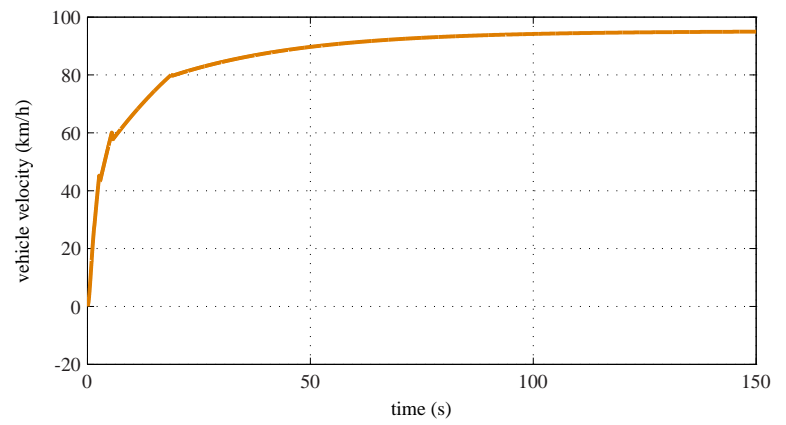

Figure 13: Vehicle velocity for the complete vehicle model with automatic transmission controller

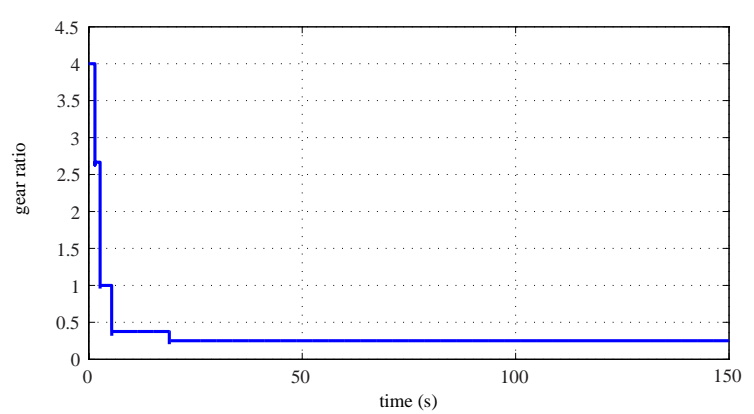

(a)

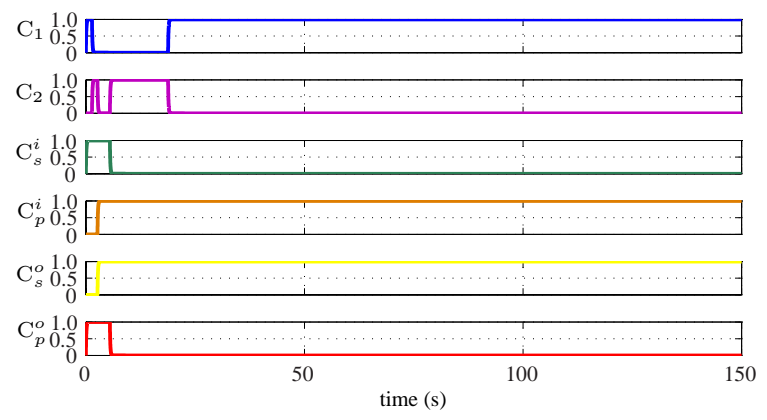

(b)

Figure 14: (a) Speed ratio; and (b) Normalized clutch pressure signals for the complete vehicle model with automatic transmission controller

[3] Oshkosh Truck Corporation, "Oshkosh Demonstrates ProPulse, the First Electric Hybrid-Drive Heavy Defense Truck," News Release, February 27, Business Wire, 2002. [Online]. Available: http://www.thefreelibrary.com/Oshkosh+Demonstrates+ProPulse\%2c+the+First+Electric+HybridDrive+Heavy...-a083328305

[4] J. Thompson, The Diesel-Electric Hybrid HEMTT A3 Lean by OSHKOSH, Diesel Power, July 1, 2011. 
[Online]. Available: http://www.trucktrend.com/cool-trucks/1107dp-diesel-electric-hybrid-hemtt-oskosha3/

[5] N. Elflein, Drive System for the World's Largest Dump Truck, Pictures of the Future, The Magazine for Research and Innovation, August 11, 2014. [Online]. Available: http://www.siemens.com/innovation/en/ home/pictures-of-the-future/mobility-and-motors/electric-mobility-world-s-largest-dump-truck.html

[6] C. Boulder, Sales of Electric Trucks and Buses Are Expected to Reach Nearly 160,000 Annually by 2023, According to Navigant Research, Info Release, January 29, 2015. [Online]. Available: http://www.businesswire.com/news/home/20150129005166/en/Sales-Electric-TrucksBuses-Expected-Reach-160000\#.VPmhii5mDxc

[7] S. Lyden, The State of All-Electric Trucks in the U.S. Medium-Duty Market, Green Fleet, January/February, 2014. [Online]. Available: http://zerotruck.com/wp-content/downloads/GRN_medium.pdf

[8] J. Knowles, D. Corne, and K. Deb, Multiobjective Problem Solving from Nature: From Concepts to Applications. Springer-Verlag, Berlin Heidelberg, 2008.

[9] A. Sorniotti, T. Holdstock, G. Pilone, F. Viotto, S. Bertolotto, M. Everitt, R. Barnes, B. Stubbs, and M. Westby, "Analysis and simulation of the gearshift methodology for a novel two-speed transmission system for electric powertrains with central motor," Proceedings of the Institution of Mechanical Engineers, Part D: Journal of Automobile Engineering, no. 226, pp. 915-929, 2012.

[10] Q. Ren, D. Crolla, and A. Morris, “Effect of transmission design on electric vehicle performance." Dearborn, USA: Proc IEEE Vehicle Power and Propulsion Conference (VPPC), 2009.

[11] A. Morozov, K. Humphries, T. Zou, S. Martins, and J. Angeles, "Design and optimization of a drivetrain with two-speed transmission for electric delivery step van." December, Florence, Italy: IEEE International Electric Vehicle Conference IEVC 2014, 2014.

[12] A. Morozov, , K. Humphries, T. Rahman, T. Zou, and J. Angeles, "Drivetrain for the class-4 electric truck: Problem definition and performance evaluation criteria," 2016, in preparation.

[13] A. Morozov, , K. Humphries, T. Zou, T. Rahman, and J. Angeles, "Drivetrain analysis and optimization for a two-speed class-4 electric truck," 2016, in preparation.

[14] A. Morozov, T. Zou, S. Rahimi, Y. Setiawan, J. Angeles, and B. Boulet, "Design and gear shift control of a modular swift-shift multi-speed transmission for EVs." Brussels, Belgium: Proceedings of European Battery, Hybrid and Fuel Cell Electric Vehicle Congress EEVC, December 1-4 2015. 


\section{Authors}

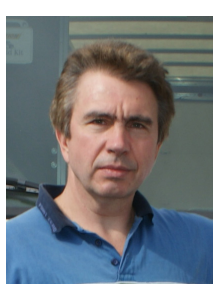

Alexei Morozov, Eng., Ph.D., is Research Engineer at the Centre for Intelligent Machines, McGill University (McGillAutomotive Partnership Canada project), and a Lecturer in the Department of Mechanical Engineering. Dr. Morozov graduated from Bauman Technical University, Moscow, in 1983, in computer science, and received a Ph.D. degree from the Graduate School of Mechanical Engineering Research Institute of Russian Academy of Sciences in Moscow in 1990, in robotics. Dr. Morozov is a registered Professional Engineer in the province of Québec, his research areas including robotics, design and optimization of drivetrains for electric vehicles, and X-by-wire technology.

Ting Zou, Ph.D., is a Postdoctoral Fellow working with Prof. Angeles at the Centre for Intelligent Machines, McGill University. Dr. Zou obtained a B.Sc. in electrical engineering from Xi'an Jiaotong University in 2005, a M.Sc. in automatic control engineering from Xi' an Jiaotong University in 2008, and a $\mathrm{PhD}$ degree in mechanical engineering from McGill University in 2013. Her research interests include design and optimization of robotic systems, automotive transmission system design and optimization.

Mir Saman Rahimi Mousavi received the B.Sc. and M.Sc. degrees in mechanical engineering from Khajeh Nasireddin Toosi University and Sharif University of Technology, Tehran, Iran, in 2008 and 2010, respectively, and is currently pursuing the Ph.D. degree in electrical and computer engineering at McGill University, Montreal, QC, Canada. His research interests include optimal control of electric and hybrid vehicle driveline, robust and nonlinear control of mechatronic systems, and dynamical modeling of multibody systems.
Jorge Angeles (SM'90-F'06) received the Dipl.Eng. degree in electromechanical engineering and the M.Eng. degree in mechanical engineering from Universidad Nacional Autónoma de México, Mexico City in 1969 and 1970, respectively, and the $\mathrm{Ph} . \mathrm{D}$. degree in applied mechanics from Stanford University, in 1973. In 1984, Angeles joined the Department of Mechanical Engineering, McGill University, Montreal, where, in 1985, he became a founding member of the Centre for Intelligent Machines and is currently James McGill Professor of Mechanical Engineering, and the Founder and Director of the Robotic Mechanical Systems Laboratory. Dr. Angeles is a Fellow of the RSC, The Academies of Arts, Humanities and Sciences of Canada. $\mathrm{He}$ is one of the 12 honorary members of IFToMM, the International Federation for the Promotion of Mechanism and Machine Science. He is a Fellow of the Canadian Society for Mechanical Engineering and the American Society of Mechanical Engineers. His professional registration as an Engineer includes Quebec, Mexico, and Germany.

Benoit Boulet, Eng., Ph.D., SMIEEE, is Associate Dean (Research \& Innovation) of the Faculty of Engineering at McGill University and an Associate Professor in the Department of Electrical and Computer Engineering. Professor Boulet obtained a Bachelor's degree from Université Laval in 1990, a Master of Engineering degree from McGill University in 1992, and a Ph.D. degree from the University of Toronto in 1996, all in electrical engineering. $\mathrm{He}$ is a registered Professional Engineer in the province of Québec. Professor Boulet's research areas include the control of biomedical systems, green energy systems, robust industrial process control and robust vehicle control. 